\title{
Avances en el tratamiento del juego patológico
}

\author{
Echeburua E. ${ }^{1}$, Fernandez-Montalvo, J. ${ }^{2}$, Baez C. ${ }^{3}$
}

(1) Departamento de Personalidad, Evaluación y Tratamientos Psicológicos. Facultad de Psicología. Universidad del País Vasco. E-mail: ptpodece@ss.ehu.es (2) Departamento de Psicología y Pedagogía. Universidad Pública de Navarra

(3) Centro de Salud Mental de Rentería. Servicio Vasco de Salud.

\begin{abstract}
Resumen
En este artículo se lleva a cabo una revisión bibliográfica sobre los avances en el tratamiento de la ludopatía. Los estudios referidos a la terapia del juego patológico prueban que este cuadro clínico responde bien a los tratamientos ofertados. No obstante, la terapia de elección parece ser el control de estímulos y la exposición en vivo con prevención de respuesta, seguida por una intervención cognitivo-conductual en prevención de recaídas. Se comentan las implicaciones de esta revisión para la práctica clínica y para las investigaciones futuras en este campo.
\end{abstract}

Palabras clave: Juego patológico. Tratamientos psicológicos. Tratamientos farmacológicos. Prevención de recaídas.

\section{Summary}

This paper deals with a review of the literature on new developments in treatment for pathological gambling. Reviewed studies about treatment of pathological gambling show that this disorder improves significantly with proposed therapies. However, the choice treatment appears to be stimulus control and in vivo exposure with response prevention, followed by a cognitive-behavioural intervention in relapse prevention. The relevance of this review for clinical practice and future research in this field is commented upon.

Key words: Pathological gambling. Psychological treatment. Pharmacological treatment. Relapse prevention.

\section{INTRODUCCIÓN}

L a ludopatía es un trastorno mental que aparece clasificado por primera vez como una entidad nosológica y con unos criterios diagnósticos específicos en el DSM-III (American Psychiatric Association, 1980). En la actualidad se halla categorizado en el DSM-IV (American Psychiatric Association, 1994) como un trastorno del control de los impulsos no clasificado en otros apartados, junto con la cleptomanía, la piromanía, el trastorno explosivo intermitente, etc. Las características generales de estos trastornos son las siguientes: el fracaso en resistir el impulso, la sensación creciente de tensión o activación antes de llevar a cabo el acto y la experiencia de placer, gratificación o alivio en el momento de consumarlo. En concreto, el juego patológico figura como un fracaso crónico y progresivo en resistir los impulsos a jugar, de los que derivan conductas de juego que interfieren negativamente en la consecución de los objetivos personales, familiares y/o profesionales (Becoña, 1996; Robert y Botella, 1995).

En nuestro país, la tasa de prevalencia de la ludopatía oscila entre el $2 \%$ y el $3 \%$ de la población adulta (Becoña, 1993; Becoña y Fuentes, 1994; Irurita, 1996; Legarda, Babio y Abreu, 1992; Tejeiro, 1996) (tabla 1). El trastorno es mucho más frecuente en hombres que en mujeres, pero éstas son mucho más reacias a buscar ayuda terapéutica por la censura social existente. A diferencia de otras conductas adictivas, el juego patológico se distribuye por todas las clases sociales y por todas las edades. No obstante, la edad de acceso al juego ha descendido en los últimos años. De hecho, cada vez son más los adolescentes que acuden a consulta por problemas de juego (Báez y Echeburúa, 1995).

Los ludópatas, al menos en España, muestran una dependencia fundamentalmente a las máquinas tragaperras, ya sea sólo a éstas o en combinación con otros juegos. La dependencia en exclusiva a otros juegos de azar es mucho menor (Báez, Echeburúa y Fer- 


\section{TABLA 1: ESTUDIOS EPIDEMIOLÓGICOS REALIZADOS EN ESPAÑA}

\section{AUTORES Y AÑO}

Cayuela, 1990

Becoña, 1991

Legarda, Babio y Abreu, 1992

Becoña y Fuentes, 1994

Irurita, 1996

Tejeiro, 1996
PROCEDENCIA

Cataluña

Galicia

Sevilla

Galicia

Andalucía

Algeciras
N

1.230

1.615

598

1.028

4.977

419
JUGADORES
PATOLÓGICOS

-

$1,73 \%$

$1,7 \%$

$1,4 \%$

$1,8 \%$

$1,9 \%$
JUGADORES

PROBLEMÁTICOS

$2,5 \%$

$1,6 \%$

$5,2 \%$

$2,0 \%$

$4,4 \%$

$3,8 \%$
nández-Montalvo, 1994). Este hecho no es fruto de la casualidad, sino que obedece a una serie de aspectos psicológicos implicados en el funcionamiento de este tipo de máquinas (Echeburúa, 1992): su amplia difusión, el importe bajo de las apuestas con posibilidad de ganancias proporcionalmente cuantiosas, la brevedad del plazo transcurrido entre la apuesta y el resultado, la manipulación personal de la máquina que genera una cierta ilusión de control, las luces intermitentes de colores que, junto con la música y el tintineo estrepitoso de las monedas cuando se gana, suscitan una tensión emocional y una gran activación psicofisiológica, etc.

Ya desde su primera descripción como un trastorno mental en el DSM-I/I (APA, 1980), la ubicación nosológica de este cuadro clínico ha sido objeto de discusión. De hecho, hoy por hoy no existe todavía un acuerdo sobre su clasificación. Mientras que las nosología psiquiátricas vigentes -DSM-IV (APA, 1994) e ICD-10 (Organización Mundial de la Salud, 1992)- lo conceptualizan como un trastorno del control de los impulsos, la mayor parte de los autores lo consideran como una adicción sin droga. En realidad, la sintomatología central de los ludópatas coincide en gran parte con la de los alcohólicos y drogodependientes. En este sentido, se ha propuesto recientemente la clasificación del juego patológico como una adicción psicológica (Echeburúa y Corral, 1994).

Desde una perspectiva psicopatológica, los trastornos del estado de ánimo (depresión e hipomanía, fundamentalmente) y las conductas adictivas están asociados con frecuencia al juego patológico. Así, por ejemplo, en la muestra de 64 jugadores patológicos de Báez et al. (1994) un 94\% de los sujetos manifestaba algún grado de sintomatología depresiva. Asimismo en el estudio de McCormick, Russo, Ramírez y Taber (1984) con 60 jugadores patológicos, había un $76 \%$ de casos de depresión y un 38\% de hipomanía.

Por otra parte, la tasa de prevalencia del consumo abusivo de alcohol u otras drogas entre los jugadores patológicos oscila alrededor del 15\% (Lesieur, Blume y Zoppa, 1986; Lesieur y Heineman, 1988; RodríguezMartos, 1987, 1989), pero en algunos estudios esta tasa es aún mayor (Báez et al., 1994; Ramírez, McCormick, Russo y Taber, 1983). El consumo de sustancias adictivas puede responder a motivaciones distintas, tales como la potenciación de la estimulación y del placer, el enfrentamiento a las vivencias del juego, el olvido de las pérdidas y/o la sustitución de la adicción (González, 1989).

Como ocurre en las conductas adictivas, el impacto del juego patológico va más allá del paciente identificado. En concreto, la familia, los amigos y el ambiente laboral están profundamente afectados por la problemática del juego del sujeto (McCormick y Ramírez, 1988). De hecho, el deterioro puede extenderse en algunos casos a la pareja del jugador, en forma de aumento de la bebida y del tabaco, de trastornos de la conducta alimentaria, de gastos impulsivos sin control, etc. (Lorenz y Yaffee, 1988).

En definitiva, el jugador patológico se caracteriza por una dependencia emocional del juego, una pérdida de control y una interferencia negativa en el funcionamiento normal en la vida cotidiana (Echeburúa y Báez, 1994).

\section{TRATAMIENTO DEL JUEGO PATOLÓGICO}

Debido a que inicialmente el juego patológico se ha considerado como una adicción, se han ensayado, especialmente en los primeros momentos, técnicas terapéuticas que han mostrado su utilidad en el control del alcoholismo y de la drogadicción (Becoña, 1996; Lester, 1980). En concreto, los tratamientos más utilizados han sido las técnicas aversivas (Barker y Miller, 1968; Seager, 1970), la reestructuración cognitiva (Bannister, 1977; Echeburúa, Báez y FernándezMontalvo, 1994), la intención paradójica (Victor y Krug, 1967), la desensibilización imaginada (McConaghy, Armstrong, Blaszczynski y Allcock, 1983, 1988), la exposición en vivo y el control de estímulos (Echeburúa et al., 1994; Greenberg y Rankin, 1982) y la terapia de grupo cognitivo-conductual (Echeburúa et al., 1994; Turón, Salgado, González y Vicente, 1990). Asimismo, 
más recientemente, se han comenzado a aplicar programas específicos de intervención psicofarmacológica (Hollander, Frenkel, DeCaria, Trungold y Stein, 1992; Saiz-Ruiz, 1992; Turón et al., 1990).

Sin embargo, si bien son abundantes las técnicas empleadas, resultan escasos los estudios publicados en los que se haya puesto a prueba la eficacia diferencial de distintas aproximaciones terapéuticas. Por otra parte, los estudios aluden generalmente a combinaciones de técnicas de las que no siempre puede aislarse el componente efectivo (Blaszczynski, 1985). Por ello, es sumamente necesaria la puesta en marcha de investigaciones rigurosas y controladas sobre el tratamiento del juego patológico.

Por lo que se refiere al objetivo terapéutico, la mayor parte de los estudios tienen como meta la abstinencia total del juego. No obstante, como ha ocurrido en el caso del alcoholismo (cfr. Echeburúa y Corral, 1988), se ha planteado la posibilidad de crear programas terapéuticos con el objetivo de lograr, si no la abstinencia total, al menos un mayor control: el juego controlado (Rosecrance, 1988). No obstante, aunque este tipo de programas resultan sugerentes, al menos para cierto tipo de jugadores, todavía no se han puesto a prueba de una forma controlada y se carece, por tanto, de un respaldo empírico a los mismos. Por ello, en la actualidad parece aconsejable considerar la abstinencia total del juego como principal objetivo terapéutico (Blaszczynski, 1995).

Las técnicas mejor estudiadas hasta la fecha son la desensibilización imaginada (variante de la desensibilización sistemática en la que las escenas no se presentan en un orden jerárquico) y la exposición en vivo con prevención de respuesta, junto con el control de estímulos (tabla 2). El resto de las técnicas, o bien han caído en desuso (por ejemplo, las técnicas aversivas), o bien remiten a estudios de casos (por ejemplo, la intención paradójica), con una escasa capacidad de generalización. Respecto a la reestructuración cognitiva, no se ha mostrado superior hasta el momento a otro tipo de técnicas más conductuales (cfr. Echeburúa et al., 1994).

En los siguientes apartados se realiza una descripción de los principales estudios llevados a cabo en relación con el tratamiento del juego patológico (tabla 3). Una revisión más exhaustiva, que incluye la descripción de otras modalidades terapéuticas, así como el trabajo realizado con programas amplios en régimen de internamiento o en Jugadores Anónimos, puede encontrarse en Echeburúa y Báez (1994).

\section{Técnicas conductuales}

\section{Desensibilización imaginada y relajación}

La utilización de técnicas reductoras de ansiedad la desensibilización imaginada, principalmente- está estrechamente relacionada con el "mecanismo de ejecución conductual" como teoría explicativa del mantenimiento del juego. La hipótesis plantea que en el Sistema Nervioso Central se establece un mecanismo neurofisiológico de ejecución de una conducta cuando dicha conducta se convierte en habitual. Este mecanismo es activado cuando la persona está en presencia de estímulos que precedieron a la realización de la conducta habitual en el pasado o cuando la persona piensa en llevar a cabo dicha conducta. Si la conducta no es completada -más aún en el caso de conductas ilícitas o generadoras de sentimientos de culpa- cuando el sujeto es estimulado para Ilevarla a cabo, el mecanismo de ejecución conductual produce un aumento de activación y, por tanto, de tensión subjetiva. El malestar producido por esta situación fuerza al sujeto a completar la conducta, incluso aunque no desee hacerlo.

En consonancia con esta hipótesis explicativa McConaghy et al. (1983) diseñaron un programa de intervención. Para ello llevaron a cabo un estudio con 20 sujetos, en el que asignaron 10 a una modalidad de desensibilización imaginada y otros 10 a una modalidad de terapia aversiva.

\section{TABLA 2: TRATAMIENTOS PRINCIPALES DEL JUEGO PATOLÓGICO}

\section{TRATAMIENTOS}

Tratamientos conductuales

Tratamientos cognitivos

Tratamientos farmacológicos
TÉCNICAS UTILIZADAS

* Desensibilización imaginada

* Relajación

* Control de estímulos

* Exposición en vivo con prevención de respuesta

* Reestructuración cognitiva

* Clomipramina

* Naltrexona

* Fluvoxamina 


\section{TABLA 3: RESULTADOS OBTENIDOS EN LOS PRINCIPALES ESTUDIOS CONTROLADOS SOBRE EL TRATAMIENTO DEL JUEGO PATOLÓGICO}

\begin{tabular}{|c|c|c|c|c|}
\hline INVESTIGADORES & TÉCNICAS & CASOS & RESULTADOS & SEGUIMIENTOS \\
\hline Greenberg y Rankin, 1982 & $\begin{array}{l}\text { Exposición con prev. de } \\
\text { respuesta + control estimular }\end{array}$ & 26 & $\begin{array}{l}19 \% \text { controlados } \\
27 \% \text { mejorados }\end{array}$ & 9 meses a 5 años \\
\hline \multirow[t]{2}{*}{ McConaghy et al., 1988} & Desensibilización imaginada & 10 & $\begin{array}{l}10 \% \text { abstinentes } \\
40 \% \text { mejorados }\end{array}$ & 1 año \\
\hline & Relajación & 10 & $\begin{array}{l}10 \% \text { abstinentes } \\
60 \% \text { mejoradolsaño }\end{array}$ & \\
\hline \multirow[t]{4}{*}{ McConaghy et al., 1991} & Exposición prolongada & 4 & $\begin{array}{l}1 \text { abstinente } \\
1 \text { controlado }\end{array}$ & 2 a 9 años \\
\hline & Relajación & 14 & $\begin{array}{l}43 \% \text { abstinentes } \\
14 \% \text { mejorados }\end{array}$ & 2 a 9 años \\
\hline & Exposición breve & 14 & $\begin{array}{l}17 \% \text { abstinentes } \\
50 \% \text { controlados }\end{array}$ & 2 a 9 años \\
\hline & Desensibilización imaginada & 33 & $\begin{array}{l}30 \% \text { abstinentes } \\
48 \% \text { mejorados }\end{array}$ & 2 a 9 años \\
\hline \multirow[t]{3}{*}{ Echeburúa et al., 1994} & $\begin{array}{l}\text { Exposición con prev. de } \\
\text { respuesta + control estimular }\end{array}$ & 16 & $69 \%$ abstinentes & 1 año \\
\hline & Terapia cognitiva grupal & 16 & $37 \%$ abstinentes & 1 año \\
\hline & $\begin{array}{l}\text { Exposición con prevención del } \\
\text { respuesta }+ \text { control estimular + } \\
\text { terapia cognitiva grupa }\end{array}$ & 16 & $37 \%$ abstinentes & 1 año \\
\hline \multirow[t]{3}{*}{ Echeburúa et al., 1999b } & $\begin{array}{l}\text { Exposición con prevención de } \\
\text { respuesta + control estimular }\end{array}$ & 23 & $52 \%$ abstinentes & 1 año \\
\hline & $\begin{array}{l}\text { Exposición con prevención de } \\
\text { respuesta }+ \text { control estimular }+ \\
\text { prevención de recaídas individual }\end{array}$ & 23 & $82 \%$ abstinentes & 1 año \\
\hline & $\begin{array}{l}\text { Exposición con prevención de } \\
\text { respuesta + control estimular + } \\
\text { prevención de recaídas grupal }\end{array}$ & 23 & $78 \%$ abstinentes & 1 año \\
\hline Sylvain y Ladouceur, 1997 & $\begin{array}{l}\text { Reestructuración cognitiva + } \\
\text { entrenamiento en solución de } \\
\text { problemas + entrenamiento en } \\
\text { habilidades sociales + prevención } \\
\text { de recaídas }\end{array}$ & 14 & $57 \%$ abstinentes & 1 año \\
\hline
\end{tabular}

En la modalidad de desensibilización imaginada los sujetos, en primer lugar, tenían que describir cuatro situaciones que les impulsaban a jugar, pero sin culminar en la conducta de juego. A continuación, se les enseñaba a tranquilizarse con un método breve de relajación. Después, los sujetos, en estado de relajación, tenían que imaginarse la primera de las cuatro situaciones, y así sucesivamente hasta que se completaban las cuatro escenas. En la modalidad de terapia aversiva los pacientes, en primer lugar, escribían en tarjetas doce frases relacionadas con aspectos excitantes de la conducta de juego y otras tres relacionadas con conductas incompatibles con el juego (por ejemplo, "ir a casa en busca de mi mujer"). A continuación, se presentaban a los pacientes las tarjetas de forma aleatoria. Los sujetos recibían choques eléctricos en los dedos cada vez que leían alguna de las doce frases relativas a la conducta de juego, pero no así cuando les correspondía leer alguna de las tres frases restantes relacionada con actividades incompatibles. Los pacientes de ambas modalidades recibieron 14 sesiones de tratamiento de 20 minutos en un período de 1 semana en régimen de internamiento.

Tras la evaluación de los pacientes al mes y al año del tratamiento con un evaluador independiente, un $70 \%$ de los sujetos tratados con desensibilización imaginada ya no mostraban un deseo vehemente de jugar ni jugaban (o lo hacían moderadamente), frente a un $20 \%$ de los sujetos tratados con terapia aversiva. 
En un estudio posterior de este mismo grupo (McConaghy et al., 1988), descartada ya la terapia aversiva, se asignaron 10 sujetos a una modalidad de desensibilización imaginada y otros 10 a una modalidad de relajación. El tratamiento se llevó a cabo en régimen de internamiento durante 1 semana. Ambas modalidades terapéuticas se mostraron efectivas para la reducción del impulso vehemente de jugar y de la conducta de juego (con tasas de éxito total o parcial del $50 \%$ al $70 \%$ de los casos al cabo de 1 año de seguimiento), así como para la disminución del grado de ansiedad, pero no hubo diferencias significativas entre ellas. Por otra parte, un alto nivel de ansiedadestado al mes de seguimiento era un predictor de fracaso terapéutico al año de seguimiento.

En un estudio más reciente de revisión de resultados terapéuticos a largo plazo (con una media de 5 años) del grupo de McConaghy (McConaghy, Blaszczynski y Frankova, 1991), se confirma la potencialidad terapéutica de la desensibilización imaginada, especialmente desde la perspectiva de costes y beneficios, que se muestra superior incluso a otras técnicas conductuales, como las técnicas aversivas, la relajación y la exposición en vivo. Lo más interesante, sin duda, de este estudio es que un programa terapéutico que implica a un único terapeuta, que dura sólo 1 semana y que no requiere más de 14 horas de tratamiento pueda tener una tasa de éxito global cercana al $66 \%$ de los casos tratados al cabo de 5 años de seguimiento. Resulta, sin embargo, cuestionable el hecho de llevarlo a cabo en régimen de internamiento, ya que la sencillez de la intervención terapéutica permite ejecutarla en un ámbito ambulatorio (Echeburúa y Báez, 1994). Por ello, los pacientes elegidos, que están necesitados de una buena motivación para el internamiento, pueden no ser representativos de los pacientes que demandan ayuda terapéutica habitualmente.

Control de estímulos y exposición en vivo con prevención de respuesta

Respecto a la exposición en vivo con prevención de respuesta y control de estímulos, en el trabajo de Greenberg y Rankin (1982) se reflejan los resultados conseguidos a lo largo de 8 años en una clínica en el tratamiento del juego patológico con técnicas de exposición o de control de estímulos. Los resultados obtenidos fueron modestos. Tras un tratamiento de 6 sesiones, con asistencia irregular, con un $50 \%$ de abandonos de la terapia y con un nivel medio de seguimiento no sistemático de 8,5 meses, de los 26 pacientes tratados 5 (el 19\%) se mantenían abstinentes y 7 (el $27 \%$ ) habían mejorado (recaían de forma intermitente), pero 14 (el 54\%) seguían jugando de forma descontrolada.

No obstante, este estudio presenta varios problemas metodológicos (Echeburúa y Báez, 1994). En pri- mer lugar, y a pesar de que los resultados se presentan de forma unitaria, hubo en realidad dos programas de tratamiento y con dos terapeutas distintos. En un programa se hacía hincapié en el control de estímulos: evitar las rutas "peligrosas", no llevar apenas dinero encima, hacer otras actividades en lugar de jugar, etc. $Y$ en el otro, el terapeuta acompañaba al paciente en las primeras sesiones en la tarea de exposición al juego, pero sin permitirle jugar, para ir retirándose progresivamente en las sesiones posteriores, a medida que el paciente era capaz de resistir la urgencia de jugar. Se utilizaban como técnicas complementarias para facilitar la resistencia a los impulsos de juego, y a modo de estrategias de autocontrol, estímulos aversivos ligeros (estirar y soltar una goma enrollada en la muñeca) y sensibilización encubierta (imaginación de una consecuencia desastrosa asociada a la conducta de juego). En uno y otro programa se solicitaba la participación en la terapia del familiar más allegado.

Y en segundo lugar, la interpretación de los resultados es equívoca. La descripción unitaria del 19\% de los casos como el porcentaje de éxito de una terapia combinada de control de estímulos y de exposición con prevención de respuesta puede enmascarar la superioridad de un método sobre otro, puesto que ambos métodos se aplicaron por separado.

Más recientemente, en el estudio de Echeburúa et al. (1994) se compara la eficacia diferencial de tres modalidades terapéuticas con el objetivo de la abstinencia del juego: a) tratamiento individual de control de estímulos y exposición en vivo con prevención de respuesta; b) terapia de grupo cognitivo-conductual; y c) una combinación de ambas modalidades terapéuticas. La importancia de este estudio radica en el tamaño de la muestra (64 jugadores), en la homogeneidad del juego (máquinas tragaperras), en la brevedad del tratamiento (6 semanas) y en que por primera vez se utiliza un grupo de control (con una duración de 6 meses) con el que se pueden comparar los resultados de los grupos terapéuticos.

En esta investigación el control de estímulos se refiere fundamentalmente al manejo del dinero y a la evitación de las situaciones o circuitos de riesgo. A medida que el tratamiento avanza, se procede a una atenuación gradual del control de estímulos y se da paso a la exposición. Con la exposición gradual en vivo y la prevención de respuesta se fuerza al sujeto a que experimente deseos de jugar y a que aprenda a resistir esos deseos de forma gradualmente más autocontrolada. Con la repetición de la exposición sistemática a las situaciones de riesgo éstas pierden la potencia de inducción al juego.

A su vez, en la terapia de grupo cognitivo-conductual los objetivos fundamentales son los siguientes: reestructurar las distorsiones cognitivas presentes, facilitar el contacto con otras personas que están en la misma situación, permitir la comunicación de las difi- 
cultades con el juego a otras personas con una problemática similar, buscar soluciones y estrategias de afrontamiento comunes y darse apoyo mutuo.

Por último, en el tratamiento combinado los pacientes reciben los dos tratamientos ya descritos. A diferencia de los sujetos pertenecientes a las dos condiciones experimentales anteriores, los pacientes acuden a la terapia dos veces por semana. Si bien la duración del tratamiento es la misma, el número total de horas es el doble que en el resto de las modalidades.

En los resultados obtenidos al finalizar el tratamiento no existen diferencias significativas entre las tres modalidades terapéuticas, que son superiores al grupo de control. Todas ellas son eficaces para detener rápidamente la conducta de juego. No obstante, en el seguimiento realizado a los 6 meses, se comienzan a observar diferencias significativas entre los grupos. En la comparación de los éxitos (abstinencia del juego) con los fracasos (recaída o abandono del tratamiento), el tratamiento individual (75\% de abstinencia) es homogéneo al tratamiento grupal $(62,5 \%$ de abstinencia) y significativamente mejor que el tratamiento combinado $(37,5 \%$ de abstinencia) y que el grupo de control (25\% de abstinencia). Por otra parte, entre el tratamiento combinado y el grupo de control no hay diferencias significativas.

Respecto a las medidas psicopatológicas evaluadas (depresión, ansiedad e inadaptación), existen diferencias significativas en la depresión y en la inadaptación, pero no en la ansiedad. En el caso de la depresión, los grupos terapéuticos son significativamente superiores al grupo de control, pero homogéneos entre sí; en el caso de la inadaptación, la única diferencia es la habida entre el tratamiento individual y el grupo de control.

En el estudio de seguimiento posterior de Echeburúa, Báez y Fernández-Montalvo (1996) se informa de los datos de esta investigación correspondientes al seguimiento de los 12 meses. Los resultados se mantienen en la misma dirección que en el control de los 6 meses, con la excepción de que ahora el tratamiento individual (68,8\% de abstinencia), además de ser superior al tratamiento combinado $(37,5 \%$ de abstinencia), es también superior al tratamiento grupal (37,5\% de abstinencia). La terapia individual (exposición en vivo con prevención de respuesta y control de estímulos) se configura, por tanto, como la opción terapéutica más efectiva.

Por otra parte, en cuanto a las variables indicadoras de éxito, en este estudio la mayor satisfacción con el tratamiento, la mayor antigüedad de la dependencia al juego y el menor nivel de neuroticismo, como variable de personalidad, eran predictores de mejores resultados a largo plazo (Báez et al., 1995).
En una investigación posterior de este mismo grupo sobre la prevención de recaídas (Echeburúa, Fernández-Montalvo y Báez, 1999a) se trató, en una primera fase del estudio, a 69 jugadores patológicos con técnicas de exposición en vivo con prevención de respuesta y control de estímulos. Los resultados obtenidos al finalizar la intervención terapéutica mostraron nuevamente la eficacia de estas técnicas para detener rápidamente la conducta de juego (100\% de abstinencia en el postratamiento). La evolución posterior de estos pacientes se describe en el apartado dedicado a la prevención de recaídas.

Además de estas investigaciones controladas, se han publicado algunos estudios de casos, que ponen a prueba las técnicas de exposición, junto con el control de estímulos, en el tratamiento del juego patológico. Los resultados obtenidos son igualmente satisfactorios (Arribas y Martínez, 1991; Báez y Echeburúa, 1995; Symes y Nicki, 1997).

En concreto, en el estudio de Arribas y Martínez (1991) se utilizaron técnicas de autoexposición con prevención de respuesta, junto con control de estímulos, en el tratamiento de cuatro pacientes dependientes (tres del bingo y uno de las máquinas tragaperras). La media de sesiones de terapia fue de 13. En ninguno de los casos se produjeron episodios de recaída en el seguimiento efectuado a los 3 y 6 meses.

El control de estímulos y la exposición en vivo con prevención de respuesta resultan también efectivos en el estudio de caso de Báez y Echeburúa (1995). El programa de tratamiento consistía en 6 sesiones, de una hora de duración y con una frecuencia semanal. Con este tratamiento, el paciente -un adolescente de 19 años, con un problema de adicción a las máquinas tragaperras- dejó de jugar al finalizar el tratamiento. La abstinencia del juego se mantenía en los períodos de seguimiento (1, 3, 6, 12 y 24 meses).

Igualmente, en el estudio de Symes y Nicki (1997), con dos pacientes aquejados de juego patológico, se produjo una mejoría significativa tras aplicar un tratamiento de exposición con prevención de respuesta.

Todo ello hace pensar que el control de estímulos y la exposición en vivo con prevención de respuesta constituyen la opción terapéutica más eficaz de la que se dispone actualmente para hacer frente a la ludopatía.

\section{Técnicas cognitivas}

La utilización de técnicas cognitivas en el tratamiento de la ludopatía ha sido llevada a cabo principalmente por el grupo de Ladouceur en Quebec (Canadá). En concreto, este grupo (Ladouceur, 1993) propone un programa multimodal en el que se pone el acento en una intervención básicamente cognitiva. Dicho programa consta de 5 componentes terapéuti- 
cos fundamentales: información sobre el juego, corrección de las creencias erróneas, entrenamiento en solución de problemas relacionados con el juego, entrenamiento en habilidades sociales y prevención de recaídas.

Sin embargo, a pesar de que los autores informan de resultados alentadores, son escasos los datos disponibles sobre la eficacia de dicho programa. Se han publicado los resultados correspondientes a 4 jugadores adolescentes a los que se aplicó este tipo de intervención (Ladouceur, Boisvert y Dumont, 1994). En el seguimiento de los 6 meses los 4 jugadores se mostraban abstinentes. Si bien estos resultados son realmente alentadores, se trata prácticamente de un estudio de caso debido al escaso tamaño de la muestra utilizada.

En otro estudio realizado por el mismo grupo (Bujold, Ladouceur, Sylvain y Boisvert, 1994) se utiliza la terapia cognitiva, junto con el entrenamiento en solución de problemas y la prevención de recaídas, en el tratamiento de 3 jugadores patológicos. Con arreglo a los resultados obtenidos, los 3 sujetos se mostraban abstinentes a los 9 meses de seguimiento. Las limitaciones de este estudio son similares a las del anterior.

En una investigación controlada posterior (Sylvain y Ladouceur, 1997) se compara la eficacia de una intervención cognitiva (reestructuración cognitiva, entrenamiento en solución de problemas, entrenamiento en habilidades sociales y prevención de recaídas) con un grupo de control. La muestra total de este estudio constaba de 29 sujetos (14 en el grupo terapéutico y 15 en el grupo de control). Los resultados en el seguimiento de los 12 meses mostraron una tasa de éxitos del $57 \%$ (9 sujetos). El porcentaje de fracasos, teniendo en cuenta tanto los abandonos como las recaídas, ascendió al $43 \%$ de los casos.

Se han publicado también algunos estudios de casos en los que se aplica la terapia racional-emotiva junto con la sensibilización encubierta y Valium (Bannister, 1977) y la terapia cognitiva de Beck (Toneatto y Sobell, 1990). Se trata, sin embargo, de estudios no controlados, que abarcan programas multimodales y muestran casos poco representativos.

En relación con estos resultados, se ha propuesto que la presencia de sesgos cognitivos en los jugadores patológicos indica la necesidad de contemplar la intervención cognitiva como parte de un tratamiento completo de la ludopatía (Griffiths, 1994; Labrador y Fernández-Alba, 1998). Sin embargo, la presencia de sesgos cognitivos en el pretratamiento se ha mostrado independiente del éxito terapéutico a los 12 meses de seguimiento (cfr. Fernández-Montalvo, Báez y Echeburúa, 1996). Por otra parte, en la investigación de Echeburúa et al. (1994), comentada previamente en el apartado dedicado a la exposición, la reestructuración cognitiva se mostró inferior a un tratamiento combinado de control de estímulos y de exposición en vivo. Por lo tanto, no parece necesaria la utilización de técnicas cognitivas en el tratamiento de la ludopatía.

No obstante, la presencia de distorsiones cognitivas en el juego patológico resulta mucho más abrumadora que en el resto de los trastornos adictivos. Asimismo, las distorsiones cognitivas pueden influir en el mantenimiento de la conducta de juego. Se requiere, por ello, un mayor número de investigaciones sobre este tema.

\section{Terapia farmacológica}

El tratamiento farmacológico se ha aplicado a los jugadores patológicos cuando éstos presentaban sintomatología depresiva, con riesgos o intentos de suicidio y ansiedad elevada (González, 1989). Así, los psicofármacos que se contemplan como aplicables al juego patológico pueden agruparse en torno a dos grandes grupos (Bombín, 1992): los ansiolíticos y los antidepresivos, que tratan de neutralizar, respectivamente, la angustia y la depresión concomitantes con el impulso patológico al juego. No obstante, en los últimos años se comienza a estudiar la posibilidad de aplicar programas psicofarmacológicos específicos en el tratamiento del juego patológico (Blaszczynski, 1993; DeCaria, Hollander, Grossman, Wong, Mosovich y Cherkasky, 1996; Saiz, 1992; Turón et al., 1990).

En un estudio de caso bien controlado (Hollander et al., 1992) se ha aplicado clomipramina, con una dosis de 125-150 mg/día, a una mujer de 31 años (con una fobia social discreta y ciertos rasgos de personalidad obsesiva) dependiente de juegos múltiples y que había sido previamente tratada, sin éxito, mediante la participación en Jugadores Anónimos y psicoterapia individual. La paciente redujo la conducta de juego considerablemente al cabo de 3 semanas de tratamiento y la interrumpió por completo al cabo de 10 semanas. La abstinencia se mantuvo, con la excepción de un episodio aislado de recaída en la semana decimoséptima de tratamiento.

En el Servicio de Psiquiatría del Hospital de Bellvitge de Barcelona, se llevó a cabo un estudio en el que se trataba a 30 pacientes con dosis progresivas de naltrexona (antagonista bloqueante de los receptores opioides) que alcanzaban unas cifras de mantenimiento equivalentes a $150 \mathrm{mg}$ en días alternos (Turón et al., 1990). La hipótesis subyacente es que el juego es una adicción no ligada al uso de sustancias y que la sobreproducción de opioides endógenos (endorfinas) desempeña un papel importante en la génesis de todas las patologías adictivas y del control de los impulsos. Los pacientes fueron controlados semanalmente durante las primeras ocho semanas y pasaron posteriormente a un control mensual en los ocho meses restantes. Al cabo de 10 meses, la tasa de 
abstinencia era de un $56 \%$ de los pacientes tratados, un $20 \%$ desde el período de evaluación previo al tratamiento y un $36 \%$ desde el inicio de la terapia. El porcentaje de recaídas fue del $20 \%$ y hubo un $17 \%$ de abandonos. El 6\% de los pacientes presentaron intolerancia a la naltrexona. No obstante, este estudio presenta una importante limitación metodológica. La ausencia de un grupo de control, con el que comparar los datos obtenidos en el grupo experimental, obliga a una interpretación cautelosa de los resultados.

Por otra parte, se intentó realizar un estudio mediante la utilización de fluvoxamina (un antidepresivo con efectos potentes sobre la transmisión serotoninérgica) en el tratamiento de jugadores patológicos (Saiz, 1992), basado en la hipótesis de la existencia de un nivel bajo de serotonina en este tipo de pacientes. Los sujetos eran asignados al azar a uno de estos dos grupos: a) fluvoxamina + psicoterapia de grupo centrada en el problema; b) placebo + psicoterapia de grupo centrada en el problema. La duración del programa era de un año y las dosis de fluvoxamina eran de 100 a 200 mg/día. Sin embargo, este estudio finalmente no se pudo terminar por las complicaciones metodológicas del diseño.

No obstante, este intento fue objeto de diversas críticas (Bombín, 1992). En primer lugar, los déficits de serotonina de los jugadores patológicos pueden ser causa, pero también consecuencia del trastorno de conducta. En este supuesto, se estaría tratando un síntoma parcial del juego. En segundo lugar, la baja proporción de serotonina también está asociada a otros síntomas clínicos que aparecen en el juego patológico (ansiedad, depresión, ideación suicida, etc.). Así, cabe preguntarse si la alteración bioquímica referida se asocia en realidad al juego patológico o más bien a los síntomas psicopatológicos que con frecuencia lo acompañan. Por otra parte, por mucho que estén presentes alteraciones bioquímicas, no se pueden soslayar los importantes factores de aprendizaje, de control de estímulos, de distorsiones cognitivas, etc., que contribuyen a mantener las conductas de juego (Bombín, 1992).

Por último, en un estudio reciente (Hollander, DeCaria, Mari, Wong, Mosovich, Grossman y Begaz, 1998), se trató a 10 jugadores patológicos con fluvoxamina. Al finalizar el tratamiento, 7 de ellos consiguieron dejar de jugar.

En resumen, si bien es prematuro obtener conclusiones definitivas, las terapias psicológicas constituyen la primera línea de tratamiento del juego patológico (Echeburúa y Báez, 1994). A modo complementario, el tratamiento farmacológico puede estar indicado sólo en aquellos casos de ludopatía con depresión grave y fuerte ansiedad, ideación suicida y/o conductas impulsivas descontroladas (Bombín, 1992; Echeburúa y Corral, 1995; Echeburúa, Corral y Salaberría, 1998).

\section{PREVENCIÓN DE LAS RECAÍDAS}

Durante los últimos años ha cobrado una especial importancia el estudio de las recaídas en las conductas adictivas. En este sentido su prevención constituye el principal reto en el tratamiento de las adicciones. De hecho, cada vez son más los estudios dirigidos a diseñar estrategias eficaces de intervención para conseguir el mantenimiento de la abstinencia (cfr. Casas y Gossop, 1993).

\section{TABLA 4: ANÁLISIS DE LAS SITUACIONES QUE PROVOCAN RECAÍDAS EN ALCOHÓLICOS, FUMADORES, HEROINÓMANOS, JUGADORES Y OBESOS (Marlatt y Gordon, 1985)}

SITUACIÓN DE LA RECAÍDA

Determinantes intrapersonales

Estados emocionales negativos

Estados físicos negativos

Estados emocionales positivos

Comprobar el control personal

Tentaciones y necesidades

TOTAL

Determinantes interpersonales

Conflictos interpersonales

Presión social

Estados emocionales positivos

TOTAL
ALCOHÓLICOS
$(\mathrm{N}=70)$

FUMADORES HEROINÓMANOS

$(\mathrm{N}=64)$

$(\mathrm{N}=129)$

JUGADORES

$(\mathrm{N}=29)$

OBESOS

$(\mathrm{N}=29) \quad \mathrm{N}=311$

TOTAL

$38 \%$

$3 \%$

$37 \%$

$2 \%$

$3 \%$

$2 \%$

$-$

$-$

$9 \%$

$11 \%$

$61 \%$

$5 \%$

$50 \%$

$18 \%$

$15 \%$

$18 \%$

$32 \%$

$3 \%$

$3 \%$

$39 \%$
$19 \%$

$9 \%$

$10 \%$

$2 \%$

$5 \%$

$45 \%$

$14 \%$

$36 \%$

$47 \%$
-
-
$16 \%$
$16 \%$
$79 \%$

$16 \%$
$5 \%$
-
$21 \%$

$33 \%$

$35 \%$

- $\quad 3 \%$

$3 \% \quad 4 \%$

- $\quad 5 \%$

$10 \% \quad 9 \%$

$46 \% \quad 56 \%$

$14 \%$

$16 \%$

$10 \% \quad 20 \%$

$28 \% \quad 8 \%$

$55 \%$

$44 \%$ 


\section{El fenómeno de la recaída}

Una revisión de los fracasos terapéuticos a largo plazo entre los alcohólicos, fumadores, heroinómanos, jugadores patológicos y obesos muestra unas tasas muy similares en estos diferentes cuadros clínicos: los dos tercios, aproximadamente, de todas las recaídas se producen en los tres primeros meses tras la terminación del tratamiento (Brown, 1987, 1989; Echeburúa y Corral, 1986; Marlatt y Gordon, 1985).

El fracaso en el mantenimiento de la abstinencia, independientemente del trastorno adictivo concreto, aparece frecuentemente asociado a las mismas tres situaciones de alto riesgo: estados emocionales negativos, conflictos interpersonales y presión social (tabla 4). Estos datos apoyan la existencia de elementos comunes subyacentes en todas las conductas adictivas $y$, por ello, la justificación del estudio de la recaída como una variable relevante en sí misma (Echeburúa, 1993).

El análisis de las recaídas en el juego patológico tiene un interés añadido en relación con el resto de los trastornos adictivos. Al no existir una sustancia tóxica que sea responsable de la dependencia física y explique, al menos hasta cierto punto, la probabilidad de recaída, en la ludopatía es posible estudiar los factores puramente psicológicos de tal fenómeno.

En este sentido, en un estudio más específico sobre las situaciones precipitantes de la recaída en el juego patológico (Fernández-Montalvo, Echeburúa y Báez, 1999, en prensa), los principales factores encontrados fueron, por orden de importancia, los siguientes: el manejo inadecuado del dinero, los estados emocionales negativos, el consumo abusivo de alcohol, la avidez por el juego (el "craving") y, por último, la presión social (tabla 5).

En concreto, casi la mitad de todas las recaídas se producen por un control inapropiado del dinero (disponibilidad de unos ingresos económicos inesperados, vigilancia excesiva del dinero por parte de la familia, terminación del pago de las deudas, etc.). El segundo

\section{TABLA 5: FACTORES PRECIPITANTES DE LA RECAÍDA EN LOS JUGADORES PATOLÓGICOS ( $\mathrm{N}=35$ )}

(Fernández-Montalvo et al., 1999, en prensa)
Factores precipitantes

Manejo inadecuado del dinero

Estados emocionales negativos

Consumo abusivo de alcohol

Avidez por el juego ("craving")

Presión social
N (\%)

$17(48,6 \%)$

$9(25,7 \%)$

$5(14,3 \%)$

$2(5,7 \%)$

$2(5,7 \%)$ factor en importancia es la presencia de estados emocionales negativos (aburrimiento/tristeza, ansiedad/estrés, ira/discusiones, etc.), que explica el $25 \%$ de las recaídas. El resto de categorías (consumo abusivo de alcohol, "craving" y presión social), si bien son también importantes, explican un porcentaje menor de los fracasos terapéuticos.

\section{El tratamiento de las recaídas}

El estudio de los factores precipitantes es fundamental para el diseño de estrategias eficaces de prevención de las recaídas. En el ámbito del juego patológico, existen pocos estudios en este sentido. En concreto, McCormick (1993) propone un plan de prevención de recaídas en la ludopatía. En este programa se incluye un análisis de los factores de predisposición a la recaída y se proporcionan a los jugadores estrategias básicas de afrontamiento, así como acciones específicas para llevar a cabo si la recaída ocurre. Sin embargo, todavía no se dispone de información acerca de su eficacia.

En una investigación reciente de Echeburúa et al. (1999a) se puso a prueba la efectividad de dos modalidades de prevención de recaídas -grupal e individualbasadas en el modelo de Marlatt y Gordon (1985), en relación con un grupo de control. Para ello, se utilizó

TABLA 6: TASA DE ÉXITOS Y RESULTADOS DE X2 EN LOS DISTINTOS MOMENTOS DE EVALUACIÓN (N=69) (Echeburúa, Fernández-Montalvo y Báez, 1999, en prensa)

\section{Evaluación}

\section{Evaluación}

Post.

1 mes

3 meses

6 meses

${ }^{*} p<0,05$

$$
\begin{array}{rr}
\text { Tratamiento } & \text { Tratamiento } \\
\text { individual } & \text { grupal } \\
\mathrm{N}(\%) & \mathrm{N}(\%)
\end{array}
$$

\author{
Tratamiento \\ grupal \\ N (\%)
}

$23(100 \%)$

$22(95,7 \%)$

$21(91,3 \%)$

20 (87\%)

** $p<0,01$
$23(100 \%)$

$21(91,3 \%)$

$21(91,3 \%)$

$20(87 \%)$
Grupo de
control
$\mathrm{N}(\%)$

$21(91,3 \%)$

4,11

$17(73,9 \%)$

$14(60,9 \%)$

5,36

$9,28 * *$

$13(56,5 \%)$ 
una muestra de 69 jugadores tratados, en una fase previa, mediante exposición en vivo con prevención de respuesta y control de estímulos. Posteriormente, una vez conseguida la abstinencia, recibían la intervención en prevención de recaídas. Los resultados obtenidos en el seguimiento de los 6 meses mostraron la superioridad de los grupos terapéuticos en relación con el grupo de control (tabla 6). En concreto, en este seguimiento los dos grupos terapéuticos eran homogéneos entre sí, con una tasa de éxitos del $87 \%$, y significativamente superiores al grupo de control (56\%) (X2=7,97; $p<0,05)$. Una descripción detallada de la guía de tratamiento utilizada para la prevención de recaídas, con el diario de sesiones correspondiente y los instrumentos de evaluación empleados, puede encontrarse en Fernández-Montalvo y Echeburúa (1997).

En el estudio de seguimiento posterior de Echeburúa, Fernández-Montalvo y Báez (1999b, en prensa) se informa de los datos de esta investigación correspondientes al seguimiento de los 12 meses. Los resultados se mantienen en la misma dirección que en el control de los 6 meses. En concreto, las tasas de éxitos en este momento son del $82 \%$ en la modalidad individual, del $78 \%$ en la grupal y del $52 \%$ en el grupo de control.

En la actualidad, a la vista de estos resultados, los esfuerzos deberían dirigirse a mejorar las intervenciones dirigidas a la prevención de recaídas, así como a respaldar empíricamente las mismas.

\section{CONCLUSIONES}

Los estudios referidos a la terapia del juego patológico prueban que este cuadro clínico responde bien a los tratamientos ofertados. Desde una perspectiva general, se han obtenido unas tasas de éxito superiores al 50\% de los casos tratados con un control mínimo de seguimiento de 6 meses. Además, esta tasa de éxito aumenta considerablemente cuando se aplican técnicas conductuales -control de estímulos y exposición en vivo con prevención de respuesta, principalmente- seguidas de una intervención cognitivoconductual en prevención de recaídas (cfr. Echeburúa et al., 1999a, 1999b). En la actualidad este tipo de técnicas constituyen, sin duda, el tratamiento de elección de la ludopatía.

El empleo de técnicas cognitivas no parece ser necesario para conseguir la abstinencia del juego. Independientemente del número elevado de distorsiones cognitivas que presentan los jugadores patológicos (cfr. Fernández-Montalvo et al., 1996), la eficacia de los tratamientos a largo plazo no aumenta cuando se incluyen este tipo de intervenciones cognitivas (cfr. Echeburúa et al., 1996).
Si bien el tratamiento psicologico es prioritario en el ámbito de la ludopatía, queda pendiente, desde una perspectiva psicofarmacológica, el estudio de las situaciones concretas en las que el empleo de medicación, de forma combinada con la terapia psicológica, aumenta la eficacia terapéutica -jugadores con un nivel elevado de impulsividad, por ejemplo-. En nuestro país, se han realizado algunos intentos en este sentido (cfr. Sáiz-Ruiz, 1992; Turón et al., 1990), pero los resultados obtenidos hasta la fecha han sido inconcluyentes.

Desde otra perspectiva, resulta de interés el tratamiento de los trastornos duales. En las investigaciones controladas sobre la terapia del juego patológico se utilizan, por razones metodológicas, jugadores puros, sin otro trastorno psicopatológico concomitante. No obstante, la experiencia clínica demuestra la existencia, en numerosas ocasiones, de comorbilidad e incluso de un diagnóstico dual en el ámbito de la ludopatía (alcoholismo, esquizofrenia y deficiencia mental, principalmente, en el eje I; trastorno antisocial y borderline de la personalidad, en el eje II). En estos casos, las técnicas terapéuticas propuestas no se muestran tan útiles como en los jugadores puros. Se requiere más investigación a este respecto.

Por último, una cuestión de gran interés es la referida a las diferencias individuales. _Necesitan todos los jugadores patológicos tratamiento para controlar su hábito? Aunque prácticamente no hay investigaciones controladas sobre la evolución espontánea de los pacientes no tratados, algunos, como ocurre también en el caso de ciertos bebedores excesivos y de ciertos fumadores, pueden dejar de jugar por sus propios medios; otros, por el contrario, no responden ni al mejor de los tratamientos disponibles (Blaszczynski, 1985). En el estudio de Echeburúa et al. (1994), por ejemplo, el $25 \%$ de los pacientes pertenecientes al grupo de control habían dejado de jugar a pesar de no haber recibido ninguna intervención terapéutica durante un período de 6 meses.

En otras palabras, la adaptación de los objetivos clínicos y de los programas de tratamiento a las necesidades individuales, así como el papel terapéutico mismo de los manuales de autoayuda (por ejemplo, Fernández-Montalvo y Echeburúa, 1997), constituyen una cuestión no resuelta que requiere una detallada investigación.

\section{REFERENCIAS BIBLIOGRÁFICAS}

American Psychiatric Association (1980). Diagnostic and Statistical Manual of Mental Disorders (3rd. ed.), Washington, DC: APA. 
American Psychiatric Association (1994). Diagnostic and Statistical Manual of Mental Disorders (4th. ed.), Washington, DC: APA.

Arribas, M.P. y Martínez, J.J. (1991). Tratamiento individual de jugadores patológicos: descripción de casos. Análisis y Modificación de Conducta, 17, 255-269.

Báez, C. y Echeburúa, E. (1995). El control de estímulos y la exposición con prevención de respuesta como tratamiento psicológico de la ludopatía en un adolescente. Análisis y Modificación de Conducta, 21, 125-145.

Báez, C., Echeburúa, E. y Fernández-Montalvo, J. (1994). Características demográficas, de personalidad y psicopatológicas de los jugadores patológicos de máquinas tragaperras en tratamiento: un estudio descriptivo. Clínica y Salud, 5, 289-305.

Báez, C., Echeburúa, E. y Fernández-Montalvo, J. (1995). Variables predictoras de abandonos y recaídas en el tratamiento del juego patológico. Análisis y Modificación de Conducta, 21, 5-22.

Bannister, G. (1977). Cognitive and behaviour therapy in a case of compulsive gambling. Cognitive Therapy and Research, 1, 227-237.

Barker, J.C. y Miller, M. (1968). Aversion therapy for compulsive gambling. The Journal of Nervous and Mental Disease, 146, 285-302.

Becoña, E. (1991). The prevalence of pathological gambling in Galicia (Spain). Meeting of the Society for the Study of Gambling. London, November.

Becoña, E. (1993). El juego compulsivo en la comunidad autónoma gallega. Santiago de Compostela. Junta de Galicia.

Becoña, E. (1996). La ludopatía. Madrid. Aguilar.

Becoña, E. y Fuentes, M.J. (1994). El juego patológico en Galicia evaluado con el South Oaks Gambling Screen. Comunicación presentada en el "23rd. International Congress of Applied Psychology". Madrid, julio.

Blaszczynski, A.P. (1985). A winning bet: Treatment for compulsive gambling. Psychology Today, December, 3846.

Blaszczynski, A.P. (1993). Juego patológico: una revisión de los tratamientos. Psicología Conductual, 1, 409-440.

Blaszczynski, A.P. (1995). Cognitive and behavioral therapies for pathological gambling. Journal of Gambling Studies, 11, 195-220.

Bombín, B. (1992). Enfoques farmacológicos. Serotonina y ludopatía. En B. Bombín (Ed). El Juego de Azar. Patología y Testimonios. Valladolid. Junta de Castilla y León.

Brown, R.I.F. (1987). Classical and operant paradigms in the management of gambling addictions. Behavioural Psychotherapy, 15, 111-122.

Brown, R.I.F. (1989). Relapses from a gambling perspective. En M. Gossop. Relapse and addictive behaviour. New York. Routdge.

Bujold, A., Ladouceur, R., Sylvain, C. y Boisvert, J.M. (1994). Treatment of pathological gamblers: an experimental study. Journal of Behavior Therapy and Experimental Psychiatry, 25, 275-282.
Casas, M. y Gossop, M. (1993). Recaída y prevención de recaídas. Barcelona. Citrán.

Cayuela, R. (1990). Characteristics and situation of gambling addiction in Spain: Epidemiological and clinical aspects. Eight International Conference on Risk and Gambling. Londres.

DeCaria, C.M., Hollander, E., Grossman, R., Wong, C.M., Mosovich, S.A. y Cherkasky, S. (1996). Diagnosis, neurobiology and treatment of pathological gambling. Journal of Clinical Psychiatry, 57, 80-84.

Echeburúa, E. (1992). Psicopatología, variables de personalidad y vulnerabilidad psicológica al juego patológico. Psicothema, 4, 7-20.

Echeburúa, E. (1993). Las conductas adictivas: _una ruta común desde el "crack" al juego patológico?. Psicología Conductual, 1, 321-337.

Echeburúa, E. y Báez, C. (1994). Tratamiento psicológico del juego patológico. En J.L. Graña (Ed.). Conductas adictivas: teoría, evaluación y tratamiento. Madrid. Pirámide.

Echeburúa, E., Báez, C. y Fernández-Montalvo, J. (1994). Efectividad diferencial de diversas modalidades terapéuticas en el tratamiento psicológico del juego patológico. Análisis y Modificación de Conducta, 20, 617-643.

Echeburúa, E., Báez, C. y Fernández-Montalvo, J. (1996). Comparative effectiveness of three therapeutic modalities in the psychological treatment of pathological gambling: long-term outcome. Behavioural and Cognitive Psychotherapy, 24, 51-72.

Echeburúa, E. y Corral, P. (1986). Prevención de la recaída en las conductas adictivas: estrategias de intervención. Drogalcohol, 11, 16-24.

Echeburúa, E. y Corral, P. (1988). El objetivo del beber controlado en el tratamiento del alcoholismo: perspectivas y limitaciones. Psicopatología, 8, 17-28.

Echeburúa, E. y Corral, P. (1994). Adicciones psicológicas: más allá de la metáfora. Clínica y Salud, 5, 251-258.

Echeburúa, E. y Corral, P. (1995). Interacciones entre la terapia de conducta y los tratamientos psicofarmacológicos. Boletín de Psicología, 46, 95-113.

Echeburúa, E., Corral, P. y Salaberría, K. (1998). Terapia de conducta y tratamientos psicofarmacológicos. En M.A. Vallejo (Ed.). Manual de terapia de conducta (vol. 1). Madrid. Dykinson.

Echeburúa, E., Fernández-Montalvo, J. y Báez, C. (1999a). Prevención de recaídas en la terapia del juego patológico: eficacia diferencial de tratamientos. Análisis y Modificación de Conducta, 25, 375-403.

Echeburúa, E., Fernández-Montalvo, J. y Báez, C. (1999b, en prensa). Relapse prevention in the treatment of slotmachine pathological gambling: long-term outcome. Behavior Therapy.

Fernández-Montalvo, J., Báez, C. y Echeburúa, E. (1996). Distorsiones cognitivas de los jugadores patológicos de máquinas tragaperras en tratamiento: un análisis descriptivo. Cuadernos de Medicina Psicosomática y Psiquiatría de Enlace, 37, 13-23. 
Fernández-Montalvo, J. y Echeburúa, E. (1997). Manual práctico del juego patológico. Ayuda para el paciente y guía para el terapeuta. Madrid. Pirámide.

Fernández-Montalvo, J., Echeburúa, E. y Báez, C. (1999, en prensa). Las recaídas en el juego patológico: un estudio de las situaciones precipitantes. Revista de Psicopatología y Psicología Clínica

González, A. (1989). Juego Patológico: Una Nueva Adicción. Madrid. Tibidabo.

Greenberg, D. y Rankin, H. (1982). Compulsive gamblers in treatment. British Journal of Psychiatry, 140, 364366.

Griffiths, M.D. (1994). The role of cognitive bias and skill in fruit machine gambling. British Journal of Psychology, 85, 351-369.

Hollander, E., DeCaria, C.M., Mari, E., Wong, C.M., Mosovich, S., Grossman, R. y Begaz, T. (1998). Short-term single-blind fluvoxamine treatment of pathological gambling. American Journal of Psychiatry, 155, 17811783.

Hollander, E., Frenkel, M., DeCaria, C., Trungold, S. y Stein, D.J. (1992). Treatment of pathological gambling with clomipramine. American Journal of Psychiatry, 149, 710-711.

Irurita, I.M. (1996). Estudio sobre la prevalencia de los jugadores de azar en Andalucía. Sevilla. Comisionado para la Droga, Consejería de Asuntos Sociales, Junta de Andalucía.

Labrador, F.J. y Fernández-Alba, A. (1998). Juego patológico. En M.A. Vallejo (Ed.). Manual de terapia de conducta (vol. 2). Madrid. Dykinson.

Ladouceur, R. (1993). Aspectos fundamentales y clínicos de la psicología de los juegos de azar y de dinero. Psicología Conductual, 1, 361-374.

Ladouceur, R., Boisvert, J.M. y Dumont, J. (1994). Cognitive-behavioral treatment for adolescent pathological gamblers. Behavior Modification, 18, 230-242.

Legarda, J.J., Babio, R. y Abreu, J.M. (1992). Prevalence estimates of pathological gambling in Seville (Spain). British Journal of Addictions, 87, 767-770.

Lesieur, H.R., Blume, S.B. y Zoppa, R.M. (1986). Alcoholism, drug abuse and gambling. Alcoholism: Clinical and Experimental Research, 10, 33-38.

Lesieur, H.R. y Heineman, M. (1988). Pathological gambling among youthful multiple substance abusers in a therapeutic community. British Journal of Addictions, 83, 765-771.

Lester, D. (1980). The treatment of compulsive gambling. The International Journal of the Addictions, 15, 201-206.

Lorenz, V.C. y Yaffee, R.A. (1988). Pathological gambling. Psychosomatic, emotional and marital difficulties as reported by the spouse. Journal of Gambling Behavior, 4, 13-26.

Marlatt, G.A. y Gordon, J.R. (Eds.) (1985). Relapse prevention: Maintenance strategies in addictive behavior change. New York. Guilford Press.
McConaghy, N., Armstrong, M.S., Blaszczynski, A. y Allcock, C. (1983). Controlled comparison of aversive therapy and imaginal desensitization in compulsive gambling. British Journal of Psychiatry, 142, 366372.

McConaghy, N., Armstrong, M.S., Blaszczynski, A. y Allcock, C. (1988). Behavior completion versus stimulus control in compulsive gambling. Implications for behavioral assessment. Behavior Modification, 12, 371384

McConaghy, N., Blaszczynski, A. y Frankova, A. (1991). Comparison of imaginal desensitisation with other behavioural treatments of pathological gambling. A two-to nine-year follow-up. British Journal of Psychiatry, 159, 390-393.

McCormick, R.A. (1993). La selección de tratamientos para los jugadores patológicos basados en factores clínicamente significativos. Psicología Conductual, 1, 441454.

McCormick, R.A. y Ramírez, L.F. (1988). Pathological gambling. En J.G. Howells (Ed.). Modern perspectives in psychosocial pathology, New York. Brunner/Mazel Inc.

McCormick, R.A., Russo, A., Ramirez, L. y Taber, J. (1984). Affective disorders among pathological gamblers seeking treatment. American Journal of Psychiatry, 141, 215-218.

Organización Mundial de la Salud. (1992). CIE-10. Trastornos mentales y del comportamiento. Madrid. Meditor.

Ramírez, L.F., McCormick, R.A., Russo, A.M. y Taber, J.L. (1983). Patterns of substance abuse in pathological gamblers undergoing treatment. Addictive Behaviors, 8, 425-428.

Robert, C. y Botella, C. (1995). Trastornos del control de impulsos: el juego patológico. En A. Belloch, B. Sandín y F. Ramos (Eds.). Manual de psicopatología. Madrid: McGraw-Hill, vol. 1_.

Rodríguez-Martos, A. (1987). El juego... otro modelo de dependencia. Aspectos comunes y diferenciales con respecto a las drogodependencias. Barcelona. APAT, Fons Informatiu, n_ 12.

Rodríguez-Martos, A. (1989). Estudio piloto estimativo de la prevalencia de juego patológico entre los pacientes alcohólicos que acuden al Programa DROSS. Revista Española de Drogodependencias, 14, 265-275.

Rosecrance, J. (1988). Active gamblers as peer counselors. The International Journal of Addictions, 23, 751766

Saiz-Ruiz, J. (1992). No hagan juego, señores. Interviú, marzo de 1992, n_ 829, 24-28.

Seager, C.P. (1970). Treatment of compulsive gamblers by electrical aversion. British Journal of Psychiatry, 117, 545-553.

Sylvain, C. y Ladouceur, R. (1997). Cognitive and behavioral treatment of pathological gambling: a controlled study. Journal of Consulting and Clinical Psychology, 65 , 727-732. 
Symes, B.A. y Nicki, R.M. (1997). A preliminary consideration of cue-exposure, response-prevention treatment of pathological gambling behaviour: two cases studies. Journal of Gambling Studies, 13, 145-157.

Tejeiro, R. (1996). Estudio sobre el juego de azar en Algeciras. Prevalencia y características sociodemográficas. Algeciras. JARCA.

Toneatto, T. y Sobell, L.C. (1990). Pathological gambling treated with cognitive behaviour therapy: a case report. Addictive Behaviors, 15, 497-501.
Turón, J.V., Salgado, P., González, A. y Vicente, P. (1990). Tratamiento farmacológico del juego patológico. Actas de la XV Reunión de la Sociedad Española de Psiquiatría Biológica (Córdoba), 368-375.

Victor, R. y Krug, C. (1967). Paradoxical intention in the treatment of compulsive gambling. American Journal of Psychotherapy, 21, 808-814. 